\title{
The stroke mothership model survived during COVID-19 era: an observational single-center study in Emilia-Romagna, Italy
}

\author{
Andrea Zini ${ }^{1} \cdot$ Michele Romoli $^{1,2}\left(\mathbb{D} \cdot\right.$ Mauro Gentile $^{1} \cdot$ Ludovica Migliaccio $^{1} \cdot$ Cosimo Picoco $^{3} \cdot$ Oscar Dell'Arciprete $^{3}$. \\ Luigi Simonetti ${ }^{4}$. Federica Naldi ${ }^{1}$ - Laura Piccolo ${ }^{1}$ - Giovanni Gordini ${ }^{3}$. Francesco Tagliatela ${ }^{4}$. Vincenzo Bua ${ }^{3}$. \\ Luigi Cirillo $^{5,6}$ - Ciro Princiotta ${ }^{5}$. Carlo Coniglio ${ }^{3}$ - Carlo Descovich ${ }^{7}$. Pietro Cortelli ${ }^{8,9}$
}

Received: 10 July 2020 / Accepted: 23 September 2020 / Published online: 8 October 2020

(C) The Author(s) 2020

\begin{abstract}
Introduction A reduction of the hospitalization and reperfusion treatments was reported during COVID-19 pandemic. However, high variability in results emerged, potentially due to logistic paradigms adopted. Here, we analyze stroke code admissions, hospitalizations, and stroke belt performance for ischemic stroke patients in the metropolitan Bologna region, comparing temporal trends between 2019 and 2020 to define the impact of COVID-19 on the stroke network.

Methods This retrospective observational study included all people admitted at the Bologna Metropolitan Stroke Center in timeframes 1 March 2019-30 April 2019 (cohort-2019) and 1 March 2020-30 April 2020 (cohort-2020). Diagnosis, treatment strategy, and timing were compared between the two cohorts to define temporal trends.

Results Overall, 283 patients were admitted to the Stroke Center, with no differences in demographic factors between cohort-2019 and cohort-2020. In cohort-2020, transient ischemic attack (TIA) was significantly less prevalent than $2019(6.9 \%$ vs $14.4 \%, p=.04)$. Among 216 ischemic stroke patients, moderate-to-severe stroke was more represented in cohort-2020 (17.8\% vs $6.2 \%, p=.027)$. Similar proportions of patients underwent reperfusion $(45.9 \%$ in 2019 vs $53.4 \%$ in 2020), although a slight increase in combined treatment was detected $(14.4 \%$ vs $25.4 \%, p=.05)$. Door-to-scan timing was significantly prolonged in 2020 compared with 2019 (28.4 \pm 12.6 vs $36.7 \pm 14.6, p=.03)$, although overall timing from stroke to treatment was preserved.

Conclusion During COVID-19 pandemic, TIA and minor stroke consistently reduced compared to the same timeframe in 2019. Longer stroke-to-call and door-to-scan times, attributable to change in citizen behavior and screening at hospital arrival, did not impact on stroke-to-treatment time. Mothership model might have minimized the effects of the pandemic on the stroke care organization.
\end{abstract}

Keywords Ischemic stroke $\cdot$ Transient ischemic-attack $\cdot$ Epidemiology $\cdot$ COVID-19

Michele Romoli

michele.romoli@studenti.unipg.it; michele.romoli@ausl.bologna.it

1 IRCCS Istituto delle Scienze Neurologiche di Bologna, Department of Neurology and Metropolitan Stroke Center, "C.A. Pizzardi" Maggiore Hospital, Largo Nigrisoli 2, 40133 Bologna, Italy

2 Neurology Clinic, University of Perugia, Perugia, Italy

3 Department of Anaesthesia, Intensive Care and Emergency Medical Services, Maggiore Hospital, Bologna, Italy

4 Neuroradiology Unit, Maggiore Hospital, IRCCS Istituto delle Scienze Neurologiche di Bologna, Bologna, Italy
5 Neuroradiology Unit, Bellaria Hospital, IRCCS Istituto delle Scienze Neurologiche di Bologna, Bologna, Italy

6 DIMES, Department of Specialty, Diagnostic and Experimental Medicine, University of Bologna, Bologna, Italy

7 Department of Clinical Governance and Quality, Bologna Local Healthcare Authority, Bologna, Italy

8 Department of Biomedical and Neuromotor Sciences, Alma Mater Studiorum-University of Bologna, Bologna, Italy

9 IRCCS Istituto delle Scienze Neurologiche di Bologna, Bologna, Italy 


\section{Background}

The COVID-19 pandemic has forced a comprehensive reorganization of stroke networks to provide optimal care while containing the risk of transmission. COVID-free and COVIDpositive pathways have been developed, with potential impact on management of time-dependent diseases [1, 2]. Increasing reports are available to date on the variations in stroke management throughout the pandemic, with results suggesting a potential gross reduction of the incidence as well as of the ratio of eligible patients, potentially attributable to the lengthening of rescue belt [3-7]. A preliminary report from Shanghai, China, demonstrated that thrombectomies were halved during the first month of the pandemic [1]. However, the interpretation of results from following investigations has been limited by study design, with some counting scanned patients[3], and some providing no specific methods to support findings $[4,5]$.

Italy has been one of the most severely hit countries. Severe restrictions, including logistic limitations and physical distancing, might have impacted on personal belief on the need for in-person consultation, as well as on the performance of the stroke belt (personnel and facilities involved in the stroke time-dependent management). Our region, Emilia-Romagna, is one of the most affected, with more than 27,000 cases and 4000 deaths.

Here, we provide a direct comparison for stroke-code admissions, hospitalizations, and timing of rescue/treatment for ischemic stroke patients in the Bologna Metropolitan Stroke Network, comparing temporal trends between 2019 and 2020 to define differences in stroke treatment, time-dependent pathway management, and overall stroke-belt performance during the COVID-19 pandemic.

\section{Patients and methods}

\section{Design and setting}

This retrospective electronic-record-based study included all patients admitted with a stroke code at the emergency department (ED) of the Bologna Metropolitan Stroke Center, Ospedale Maggiore, between 1 March 2019 and 30 April 2019 and 1 March 2020 and 30 April 2020. The study was approved by the Local Ethics Committee, with patient consent waived given to the retrospective anonymous collection of data, according to Italian regulations. Since 2018, the Metropolitan Stroke Network was based on a direct mothership paradigm [8], to serve a population of approximately 1 million inhabitants. Twelve hospitals were reorganized, and one Comprehensive Stroke Center was established at the Maggiore Hospital. The Comprehensive Stroke Center was programmed to provide reperfusion treatments, with 5 stroke units in spoke hospitals networked with the Hub to receive and manage treated and untreated patients. The Stroke Network has been functioning since early 2018, with no changes in direct mothership model ever since. Outof-hospital rescue services can count on ambulance services and helicopter in cases of expected transport time exceeding $45 \mathrm{~min}$. In-hospital assessment is performed at arrival by the team of neurologists and dedicated stroke physicians. Advanced imaging is available $365 / 24 / 7$, with brain computerized tomography (CT) scan followed by $\mathrm{CT}$ perfusion (CTP) and CT angiogram (CTA), with tissue-based windows implemented to help in defining treatment indication when needed. During the COVID-19 pandemic, the Emergency Department (ED) planned separated pathways for COVID19-suspected vs COVID-19-negative patients, with dedicated in-hospital diagnostic facilities. COVID-19 screening included rapid nasopharyngeal swab and body temperature check. High-resolution lung CT scan was performed to all suspected patients as well as to those undergoing reperfusion strategies, in order to orient the patient towards the appropriate angiosuite in case of endovascular intervention. There was no change in brain imaging/anesthetic protocol, staff, or seniority of staff during the pandemic.

\section{Patient identification and temporal trends considered}

The March-April timeframe was selected to allow direct comparison between a period of standard workload (March-April 2019, cohort-2019) and the workload during the COVID-19 pandemic, after logistic restrictions were put into place by national and local government (March-April 2020, cohort2020). We aimed to evaluate the performance of the stroke belt, and therefore programmed the extraction of all people admitted via stroke code during the timeframe considered. Therefore, data were derived from the electronic regional health system with anonymous record linkage, for which the Internal Ethical Committee waived the need for patient informed consent. Once obtained all stroke codes activated via 118/ED call, we cross-linked data with hospital records, reviewed charts and ICD9 diagnosis, in order to extract the pool of patients suffering from an ischemic stroke, independently from treatment received. ICD9 coding for stroke and reperfusion strategies at admission and discharge were matched to define treatments provided, refine diagnosis, and avoid inclusion of mimics [9]. As a further step to confirm the robustness of the cohort, we cross-checked patients receiving treatment with records of consecutively enrolled patients in SITS database (sitsinternational.org/registries/sitsthrombolysis/, sitsinternational.org/registries/sitsthrombectomy/) and the Italian Registry of Endovascular Treatment in Acute Stroke (IRETAS).

Data regarding date of stroke, rescue belt (personnel and facilities involved in territorial emergency services), arrival in hospital, baseline National Institute of Health Stroke Scale 
score (NIHSS), stroke severity (minor 0-5; moderate 6-15; moderate to severe 16-20; severe $>20$ ), diagnostics, timing of treatment, and type of treatment (intravenous thrombolysis (IVT), endovascular thrombectomy (EVT), or both IVT + EVT) were collected and compared between the two cohorts selected.

Statistical analysis was performed with SPSS and R software. Descriptive statistics are presented for continuous variables as means and standard deviations, and were tested for normal distribution. Categorical variables are presented as counts and percentages. $\chi^{2}$ and Student's $T$ test were used for univariate inference as appropriate, with Bonferroni adjustment. Significance level was set to be 0.05 .

\section{Results}

ED admissions were 10,689 in March-April 2019 and 6897 in March-April 2020. During the 4 months considered, 283 patients $\left(n_{2019}=138, n_{2020}=145\right)$ were admitted to the Comprehensive Stroke Center with a suspected diagnosis of stroke. No differences in NIHSS, age, and gender distribution emerged between cohorts (Table 1).

TIA admitted to the ED were 65 in March-April 2019 and 38 in March-April 2020 (relative variation $=-41.5 \%$ ). Consequently, TIA admitted to the Stroke Unit were overall under-represented $(6.9 \%$ vs $14.5 \%, p=.04)$, despite similar rates of hospitalization for high risk TIA $(30.7 \%$ vs $26.3 \%, p=$ .9). Intracerebral hemorrhage remained grossly unchanged (Table 1).

Overall, 215 patients were diagnosed with ischemic stroke, 97 in cohort-2019 and 118 in cohort-2020, with higher prevalence of patients with moderate to severe stroke in cohort$2020(17.8 \%$ vs $6.2 \%, p=.027$; Table 2$)$. Similar proportions

Table 1 Characteristics of included cohorts (whole population)

\begin{tabular}{lll}
\hline & $\begin{array}{l}\text { Cohort-2019 } \\
(n=138)\end{array}$ & $\begin{array}{l}\text { Cohort-2020 } \\
(n=145)\end{array}$ \\
\hline $\begin{array}{l}\text { Female, } n(\%) \\
\text { Age, mean } \pm \text { SD }\end{array}$ & $68(49.3 \%)$ & $67(46.2 \%)$ \\
Cerebrovascular disease & $75.2 \pm 13.8$ & $71.7 \pm 18$ \\
Intracerebral hemorrhage, $n(\%)$ & $21(15.2 \%)$ & $17(11.7 \%)$ \\
Transient ischemic attack, $n(\%)$ & $20(14.5 \%)$ & $10(6.9 \%)^{*}$ \\
Ischemic stroke, $n(\%)$ & $97(70.3 \%)$ & $118(81.4 \%)^{*}$ \\
NIHSS, mean \pm SD & $7.6 \pm 7.1$ & $9.2 \pm 7.6$ \\
Month & & \\
March, $n(\%)$ & $62(44.9 \%)$ & $72(49.7 \%)$ \\
April, $n(\%)$ & $76(55.1 \%)$ & $73(50.3 \%)$ \\
\hline
\end{tabular}

$* p<0.05$

NIHSS National Institute of Health Stroke Scale
Table 2 Demographic and clinical details, treatment and timings and timings for patients admitted with acute ischemic stroke

\begin{tabular}{|c|c|c|}
\hline & Cohort-2019 $(n=97)$ & Cohort-2020 $(n=118)$ \\
\hline Female, $n(\%)$ & $54(55.1 \%)$ & $56(47.5 \%)$ \\
\hline Age, mean $\pm \mathrm{SD}$ & $76.6 \pm 13.7$ & $72.9 \pm 16.6$ \\
\hline NIHSS, mean \pm SD & $8.9 \pm 7.2$ & $9.5 \pm 7.4$ \\
\hline \multicolumn{3}{|l|}{ Stroke severity, $n(\%)$} \\
\hline Minor stroke & $2(2.1 \%)$ & $0(0 \%)$ \\
\hline Moderate stroke & $78(80.4 \%)$ & $88(74.6 \%)$ \\
\hline Moderate to severe & $6(6.2 \%)$ & $21(17.8 \%)^{*}$ \\
\hline Severe & $11(11.3 \%)$ & $9(7.6 \%)$ \\
\hline Reperfusion, $n(\%)$ & $44(45.4 \%)$ & $63(53.4 \%)$ \\
\hline IVT only & $23(23.7 \%)$ & $21(17.8 \%)$ \\
\hline EVT only & $7(7.2 \%)$ & $12(10.2 \%)$ \\
\hline $\mathrm{IVT}+\mathrm{EVT}$ & $14(14.4 \%)$ & $30(25.4 \%)^{*}$ \\
\hline All IVT & $37(38.1 \%)$ & $51(43.2 \%)$ \\
\hline All EVT & $21(21.6 \%)$ & $42(35.6 \%)^{*}$ \\
\hline \multicolumn{3}{|c|}{ Reperfusion timing, mean $\pm \mathrm{SD}^{\#}$} \\
\hline Stroke to call & $33.7 \pm 40.2$ & $65.5 \pm 104.3$ \\
\hline Call to rescue & $15.4 \pm 7.9$ & $12.9 \pm 5.8$ \\
\hline Rescue to door & $53.2 \pm 38$ & $44 \pm 26.6$ \\
\hline Door to scan & $28 \pm 12.6$ & $36.7 \pm 14.6^{*}$ \\
\hline Door to needle & $67.8 \pm 20.8$ & $72.6 \pm 34.3$ \\
\hline Stroke to needle & $164.5 \pm 51.8$ & $173.9 \pm 71.2$ \\
\hline Door to groin & $116.9 \pm 39$ & $118.8 \pm 55.7$ \\
\hline Groin to recanalization & $53.1 \pm 35.6$ & $66.8 \pm 43.9$ \\
\hline Door to recanalization & $170 \pm 53.4$ & $186 \pm 82.8$ \\
\hline Stroke to recanalization & $337.7 \pm 310.2$ & $361 \pm 270$ \\
\hline
\end{tabular}

$* p$ value $<0.05$

${ }^{*}$ Complete timing data available for $33 / 44$ patients in cohort-2019 and $49 / 63$ patients in cohort-2020

of patients underwent reperfusion $(2019=45.4 \%$ vs $2020=$ $53.4 \%$ ), with significant increase in combined treatment $(14.4 \%$ vs $25.4 \%, p=.05)$ and overall EVT $(21.6 \%$ vs $35.6 \%, p=.025$, Fig. 1). Stroke-to-call time was marginally longer in cohort-2020 $(65.5 \pm 104.3 \mathrm{~min}$ vs $33.7 \pm 40.2 \mathrm{~min}, p$ $=.06$ ), with higher variability in 2020 . Timing of treatment and rescue did not differ significantly between the cohorts. On the contrary, door-to-scan timing was significantly prolonged in 2020 compared with 2019 (28.4 vs $36.7 \mathrm{~min}, p=.03$ ). However, stroke-to-recanalization remained unchanged.

\section{Discussion}

This observational before-after study comparing access to reperfusion strategies and treatment in a single Comprehensive Stroke Center demonstrates that the performance of the mothership-based stroke network was preserved during the pandemic. 
all IVT

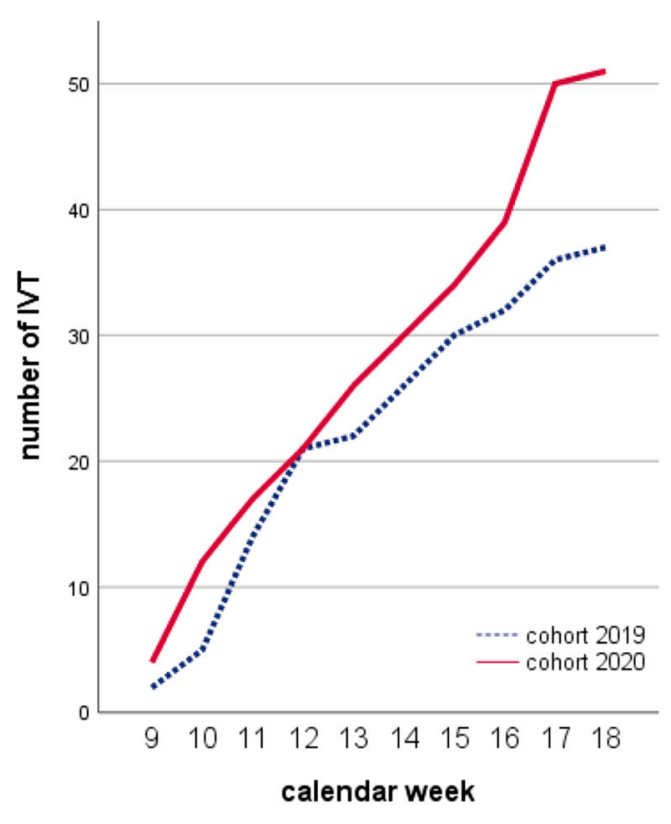

all EVT

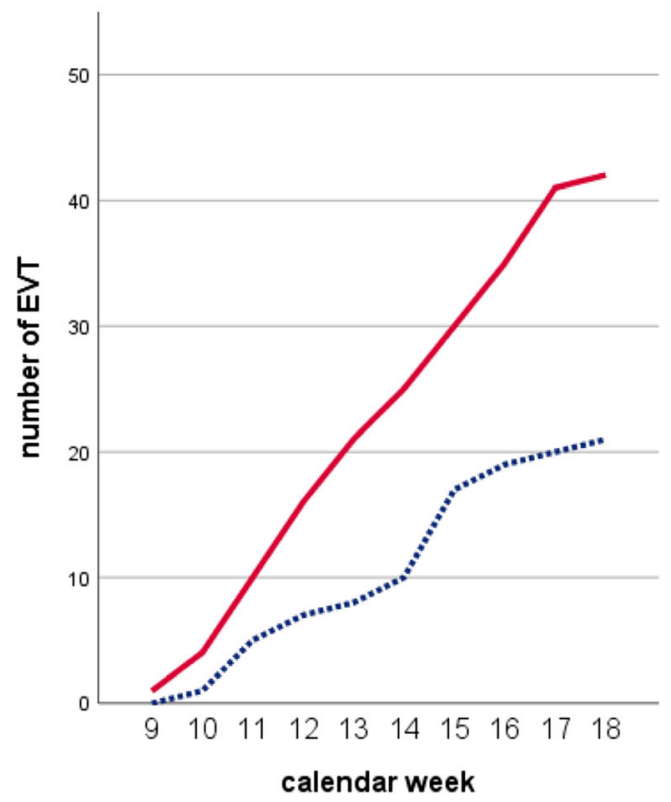

Fig. 1 Temporal trends of reperfusion treatments across weeks in 2019 and 2020 cohorts, starting from 1/3 (week 9) to 30/4 (week 18). EVT endovascular thrombectomy, IVT intravenous thrombolysis

First, we observed a consistent reduction in number of TIA and minor stroke admitted to the emergency department. Policies minimizing non-essential in-person provider-patient interactions might have impacted, as well as personal reticence to access the ED during the pandemic. If so, a consistent increase in patients searching medical attention weeks of months after TIAs or minor stroke might be predictable once the pandemic is over. For that time, our stroke care network has to meet the need of appropriate secondary prevention and rehabilitation.
Second, our results support the elasticity of a mothership paradigm, which maintained its performance over the pandemic. This is in contrast with previous studies reporting reduction in hospitalized patients and revascularization treatments [3-7], which however were based on different logistic paradigms. Although delay in emergency calls was observed, possibly reflecting a change in behavior of the population, the performance of the stroke belt was substantially preserved. The marginally longer stroke-to-call time depended on larger variability in the cohort-2020, suggesting that stroke awareness campaign might be implemented during the pandemic to reinforce a timely access to emergency services. Citizens' stroke awareness is paramount to guarantee access to treatment, and recent regional campaigns in 2014 [10] and in 2018 (https://salute.regione.emilia-romagna.it/campagne/ ictus-vedo-riconosco-chiamo) might have contributed to early recognition, and encouraged calling emergency services.

The in-hospital path was characterized by longer door-toscan time, which might be plausibly attributable to the need of a pre-screening station addressing body temperature and stratification for COVID-19. However, delay in scanning was minimal, and did not impact on stroke-to-treatment timing, which was similar to 2019. To this extent, the use of CT scan might ease and shorten the path compared with brain MRI during the pandemic.

Regarding reperfusion strategies, a significant increase in EVT was found in cohort-2020 compared with cohort-2019. Several factors might have contributed: (i) the progressive improvement of a recently developed stroke network, (ii) an improvement in skills for endovascular approach in cases of distal occlusions, (iii) an increase in stroke severity observed in cohort-2020, with potential higher rates of large vessel occlusion. The global stroke-to-treatment time was similar in 2019 vs 2020 and it might reflect the adherence to the predefined stroke network also during the COVID-19 outbreak. Overall, the boat seemed to have survived the COVID-19 storm, although inhospital path can still be improved/shortened [11].

Limitations to this study can be found in the small sample size and in the electronic-derived cohorts, which might have to some extent underestimated the prevalence of stroke. However, the paradigm seemed consistent with prospective data, since no patient was excluded according to ICD9 coding, and similar retrieve strategies were applied to both cohorts. Second, the lack of analysis of neuroimaging data limits the interpretation of the increase in EVT treatment approach, which however seems marginally significant and might well reflect the evolution of endovascular procedures towards more distal occlusions over time.

\section{Conclusion}

COVID-19 pandemic has represented and still seems a bad bargain to get the best out of. The management of stroke should 
be organized to comply with the time-dependent nature of the disease. Resources should be allocated, and mothership or dripand-ship paradigms should be critically appraised to guarantee optimal care and limit delays due to pandemic circumstances.

Acknowledgements Open access funding provided by Università degli Studi di Perugia within the CRUI-CARE Agreement.

Authors' contributions AZ, MR, MG, and LM designed and developed the study. MR performed statistical analysis and drafted the manuscript. LM and CD collected data. All authors revised and reviewed the manuscript for content.

Data availability Requests for data sharing can be directed to the corresponding author

\section{Compliance with ethical standards}

Conflict of interest The authors declare that they have no conflict of interest.

Ethical approval The protocol was approved by the Local Ethics Committee, with patient consent waived given to the retrospective anonymous collection of data, according to Italian regulations.

\section{Code availability Not applicable}

Open Access This article is licensed under a Creative Commons Attribution 4.0 International License, which permits use, sharing, adaptation, distribution and reproduction in any medium or format, as long as you give appropriate credit to the original author(s) and the source, provide a link to the Creative Commons licence, and indicate if changes were made. The images or other third party material in this article are included in the article's Creative Commons licence, unless indicated otherwise in a credit line to the material. If material is not included in the article's Creative Commons licence and your intended use is not permitted by statutory regulation or exceeds the permitted use, you will need to obtain permission directly from the copyright holder. To view a copy of this licence, visit http://creativecommons.org/licenses/by/4.0/.

\section{References}

1. Zhao J, Rudd A, Liu R (2020) Challenges and potential solutions of stroke care during the coronavirus disease 2019 (COVID-19) outbreak. Stroke 364:2019-2020. https://doi.org/10.1161/ STROKEAHA.120.029701

2. Khosravani H, Rajendram P, Notario L et al (2020) Protected code stroke: hyperacute stroke management during the coronavirus disease 2019 (COVID-19) pandemic. Stroke 2019:STROKEAHA120029838. https://doi.org/10.1161/STROKEAHA.120.029838

3. Kansagra AP, Goyal MS, Hamilton S et al (2020) Collateral effect of COVID-19 on stroke evaluation in the United States. N Engl J Med:NEJMc2014816. https://doi.org/10.1056/NEJMc2014816

4. Baracchini C, Pieroni A, Viaro F, Cianci V, Cattelan AM, Tiberio I, Munari M, Causin F (2020) Acute stroke management pathway during coronavirus-19 pandemic. Neurol Sci 41:1003-1005. https://doi.org/10.1007/s10072-020-04375-9

5. Morelli N, Rota E, Terracciano C, Immovilli P, Spallazzi M, Colombi D, Zaino D, Michieletti E, Guidetti D (2020) The baffling case of ischemic stroke disappearance from the casualty department in the COVID-19 era. Eur Neurol 83:213-215. https://doi.org/10. $1159 / 000507666$

6. Kerleroux B, Fabacher T, Bricout N et al (2020) Mechanical thrombectomy for acute ischemic stroke amid the COVID-19 outbreak. Stroke. https://doi.org/10.1161/STROKEAHA.120.030373

7. Yaghi S, Ishida K, Torres J et al (2020) SARS2-CoV-2 and stroke in a New York healthcare system. Stroke:STROKEAHA120030335. https://doi.org/10.1161/STROKEAHA.120.030335

8. Vidale S, Romoli M, Consoli D et al (2020) Bridging versus direct mechanical thrombectomy in acute ischemic stroke: a subgroup pooled meta-analysis for time of intervention, eligibility, and study design. Cerebrovasc Dis:1-10. https://doi.org/10.1159/000507844

9. Li L, Binney LE, Luengo-Fernandez R, Silver LE, Rothwell PM, on behalf of the Oxford Vascular Study (2020) Temporal trends in the accuracy of hospital diagnostic coding for identifying acute stroke: a population-based study. Eur Stroke J 5:26-35. https:// doi.org/10.1177/2396987319881017

10. Denti L, Caminiti C, Scoditti U, Zini A, Malferrari G, Zedde ML, Guidetti D, Baratti M, Vaghi L, Montanari E, Marcomini B, Riva S, Iezzi E, Castellini P, Olivato S, Barbi F, Perticaroli E, Monaco D, Iafelice I, Bigliardi G, Vandelli L, Guareschi A, Artoni A, Zanferrari C, Schulz PJ (2017) Impact on prehospital delay of a stroke preparedness campaign. Stroke 48:3316-3322. https://doi. org/10.1161/STROKEAHA.117.018135

11. Romoli M, Paciaroni M, Tsivgoulis G et al (2020) Mothership versus drip-and-ship model for mechanical thrombectomy in acute stroke: a systematic review and meta-analysis for clinical and radiological outcomes. J Stroke 22. https://doi.org/10.5853/jos.2020. 01767

Publisher's note Springer Nature remains neutral with regard to jurisdictional claims in published maps and institutional affiliations. 